\title{
A Simple and Accurate Calculator for Copper Kettle Vaporizer
}

\author{
By
}

\author{
Takao Saito, Yoshibumi Kataoka and Kenichi Iwatsuki \\ Department of Anesthesiology, Tohoku University School \\ of Medicine, Sendai
}

(Received for publication, April 13, 1961)

Although various sorts of "flow-temperature-concentration" nomograms and calculators for a copper kettle vaporizer have been introduced up to date, a presentation of a simpler and more convenient one is still desired by many anesthesiologists.

For the convenience of practical use, a compact slide rule with an ordinary cursor is presented here by the authors as a new type of calculator.

The performance characteristic of a "copper kettle vaporizer" is well expressed in the formula cited below :

$$
\begin{aligned}
& C=-\frac{a}{F_{t}} \times 100(\%)=\frac{F_{k}}{F_{t}} \times \frac{760}{760-P} \times 100(\%) \\
& P(\mathrm{mmHg}) \text { : vapor pressure of a volatile anesthetic } \\
& \text { agent contained in the copper kettle } \\
& \text { vaporizer }
\end{aligned}
$$

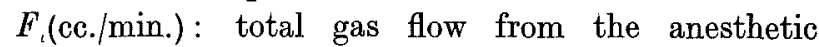

$$
\begin{aligned}
& \text { machine to the inhaler } \\
& F_{k} \text { (cc./min.): inflow of oxygen to the copper kettle } \\
& \text { vaporizer } \\
& a(\text { cc./min. }): \text { volume of an anesthetic vapor taken up } \\
& \text { by the oxygen flow through the } \\
& \text { vaporizer } \\
& C(\% \mathrm{v} / \mathrm{v}) \text { : concentration of an anesthetic vapor in } \\
& \text { the whole gas mixture }
\end{aligned}
$$

Therefore, under a certain temperature of a volatile anesthetic agent, there exists a directly proportional relationship between oxygen inflow to the copper kettle and anesthetic vapor concentration, and an inversely proportional relationship between total gas flow and anesthetic vapor concentration. The logarithmic scale was applied in this calculator.

辣藤 隆婎, 片岡 敬文, 岩月 賢一 
The basic scales are calibrated in kettle flow (cc./min. $-\boldsymbol{F}_{k}$ scale) (a) and anesthetic vapor concentration (\% V/V-C scale) (c). The sliding scale (b) is calibrated in total gas flow (cc. $/ \mathrm{min} .-F_{t}$ scale) and temperature $\left({ }^{\circ} \mathrm{C}\right)$ of various volatile anesthetic agents (chloroform, ether, fluothane and trichlorethylene) as seen in Figs. 1 and 2.

In a standard position of the calculator, $F_{k} / F_{t}$ was calibrated in the proportion of $1 / 10$. Ex. 1

Two examples of calculation are presented:

Fig. 1 illustrates how to calculate vapor concentration when the temperature of anesthetic, total gas flow and oxygen inflow to the copper kettle vaporizer are known.

If $F_{k}$ is set at $500 \mathrm{cc} / \mathrm{min} ., F_{t}$ at $5000 \mathrm{cc} . / \mathrm{min}$. and the temperature of chloroform is $20^{\circ} \mathrm{C}$, the vapor concentration of chloroform supplied to the inhaler is about $2.6 \%(V / V)$. This corresponds very well to the value directly calculated from the formula, that is $2.62 \%$. In this case it is not necessary to shift the sliding scale (b).

Ex. 2

Fig. 2 illustrates an example in which $2.3 \%(V / V)$ of ether vapor is desired to be delivered from the anesthetic machine to the inhaler. Temperature of ether is supposed to be $25^{\circ} \mathrm{C}$.

Firstly, move the cursor (d) so that the cursor line (e) corresponds to $2.3 \%$ on the $C$ scale (c).

Secondly, shift the sliding scale (b) toward left and make the line of $25^{\circ} \mathrm{C}$ of ether correspond to the cursor line (e).

Then, $F_{k} / F_{t}=1 / 100$ is obtained on the a and b scale. For example, if total flow of $5 \mathrm{l} . / \mathrm{min}$. is desired, oxygen inflow to the vaporizer should be $50 \mathrm{cc} . / \mathrm{min}$. in this case.

Instead of "diluent flow", total gas flow was used in this scale in order to make the calculator as compact as possible.

The relationship between $F_{t}$ (total flow) and $F_{d}$ (diluent flow) is well described in the formula shown below.

$$
F_{d}=F_{t}\left(1-\frac{C}{100}\right)-F_{k}
$$

Also, when $F_{k}$ and $C$ are small enough as compared with $F_{t}, F_{t}$ could be dealt as $F_{d}$ without making any significant discrepancies in calculation.

The scale was originally designed for ordinary conditions of the vaporizer in the operating room. Therefore, the calculated values in extreme conditions might neither be applicable nor true any more. For example, a condition represented by the formula " $F_{k}=F_{t}$ " could not be produced under room temperature except in the case $F_{k}=F_{t}=0$. 
T. Saito et al.
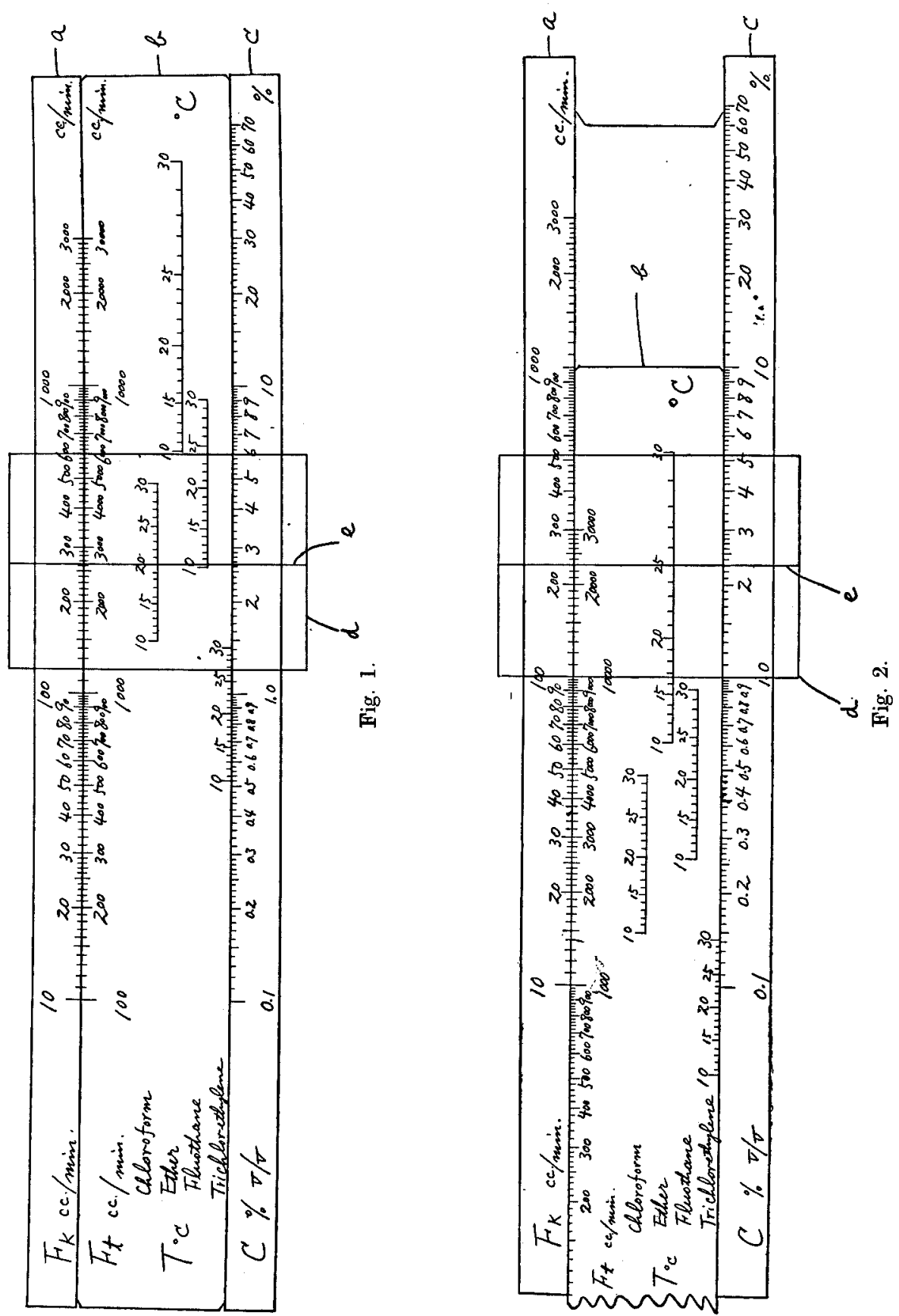

We are using this calculator with great satisfaction in clinical anesthesia and laboratory experiments. 